\title{
Monitoring Mis-Operating Conditions of Journal Bearings based on Modulation Signal Bispectrum Analysis of Vibration Signals
}

\author{
Osama Hassin ${ }^{1 *}$, Usama Haba ${ }^{1,2}$, Fengshou Gu${ }^{2}$, Andrew Ball ${ }^{2}$ \\ ${ }^{1}$ Department of mechanical, College of Engineering, University of Gharyan, Gharyan, Libya \\ ${ }^{2}$ School of Computing and Engineering, University of Huddersfield, Huddersfield, UK
}

DOI: https://doi.org/10.21467/proceedings.4.18

* Corresponding author email: osamingo@yahoo.com

\begin{abstract}
Journal bearings are widely used to undertake a wide range of operating conditions. Under high radial load, low rotating speed and thin lubrication films, asperity collisions between the journal and the bearing surfaces may occur. These self-excitations can create wideband random vibrations due to asperity contact and asperity churn. On the other hand, under low radial load and high rotating speed they can have instable operations due to oil whirls and shaft fluctuations. In addition, because of high lubricant viscosity, fluid shearing forces can be dominant, which may also result in wideband vibrations. These types of excitations together with structural responses can be coupled to produce nonlinear modulation phenomena. In this paper, modulation signal bispectrum (MSB) is used to analyses the vibration responses in order to identify the vibration signals values under different operating conditions and hence achieve condition monitoring of journal bearings. Furthermore, MSB coherences display clear regular modulating components in the high carrying frequency range due to coupling between shaft frequencies and the wideband compounds. In associating MSB-Coh. with normalising of MSB magnitude makes possibility to differentiate responses between asperity collision vibration and instability vibration. Thereby, it is possible to identify optimal operating conditions and detect abnormal operations caused by degraded lubrications or worn surfaces.
\end{abstract}

Keywords: Journal bearings, vibration condition monitoring, MSB, optimal operating conditions.

\section{Introduction}

A self-aligning spherical journal bearing has been designed for applications such as high speed fans and other similar applications. One usage of the journal bearing is to support radial load under high speed. It also designed to work at hydrodynamic lubricant regime in which thick oil film formed between the shaft and the bearing. Because of lubricant stiffness and damping coefficients, journal bearings are considered the best element for absorbing vibration, resisting shock, quietness and long life. In contrast, high radial load, low rotating speed, and weak of oil film may lead the journal bearing to work at boundary lubricant regime which causes metal-

to-metal contact.

(C) 2018 Copyright held by the author(s). Published by AIJR Publisher in Proceedings of First Conference for Engineering Sciences and Technology (CEST-2018), September 25-27, 2018, vol. 2.

This is an open access article under Creative Commons Attribution-NonCommercial 4.0 International (CC BY-NC 4.0) license, which permits any non-commercial use, distribution, adaptation, and reproduction in any medium, as long as the original work is properly cited. ISBN: $978-81-936820-6-7$ 
The idea of condition monitoring is monitoring the asset behaviour to detect, diagnose and prognoses faults and mis-operating conditions that have reached a certain symptomatic level to provide system breakdown. Vibration monitoring analysis is one of the main techniques used to diagnose and predict various defects [1]. Vibration monitoring is to break down a composite signal which generated from different vibration sources in which each source has a unique signal characteristic. Therefore, when the analyses of the journal bearing vibration signals carried out, many different sources of vibrations should be considered. The most well know external excited vibration sources of journal bearing are mechanical unbalance and misalignment. Furthermore, oil coefficients and asperity collisions also causes self-excited vibrations of journal bearing [2]. In this paper, vibration monitoring analysis is used to detect mis-operating conditions. When the machine operating conditions changes, the vibration signal changes accordingly. Modulation Signal Bispectrum (MSB) is a technique used to analyse the vibration of the composite signal which is a combination of the information and the carrier signals. This research found that MSB magnitude and MSB coherence are useful to identify vibration caused by internal and external excitations. MSB magnitude directed to vibration caused by self-excitation forces, in contrast, MSB coherence indicated to vibration caused by external-excitation forces. Finally, optimal operating conditions have been identified by coupling mean values of both MSB-Mag. and MSB-Coh. spectrums. Because the journal bearing does not contain any rotating element as a rolling bearing, vibration signals correlated to shaft rational speed and random asperity collisions.

\section{The Lubrication Regimes}

In 1846, Stribeck reported that the friction coefficient was inversely proportional to speed. Thus, he presented the characteristic curve of the coefficient of friction versus speed. Figure 6 illustrations the Stribeck curve, this shows the relationship between the coefficient of friction and bearing parameter or modulus $\eta \mathrm{N} / \mathrm{p}$, where $\eta$ is the absolute viscosity of the lubricant in $\mathrm{kg} / \mathrm{m} . \mathrm{s}, \mathrm{N}$ is the shaft speed in $\mathrm{rpm}$ and $\mathrm{p}$ is the pressure on the projected area in Pa. The Stribeck curve shows how the coefficient of friction changes with lubrication regime: boundary lubrication, mixed-film lubrication and hydrodynamic lubrication. The optimum point of the curve is when the coefficient of friction passed through a minimum point from mixed to hydrodynamic lubrication [4]. Many parameters influence the friction coefficient such as the operating condition (speed and pressure), the material properties (roughness of surface) and the viscosity of the lubricant. Later, the Stribeck curve application extended to a number of tribology component besides journal bearing. For example, ball bearing, seals and wet clutches have applied the Stribeck curve idea. 


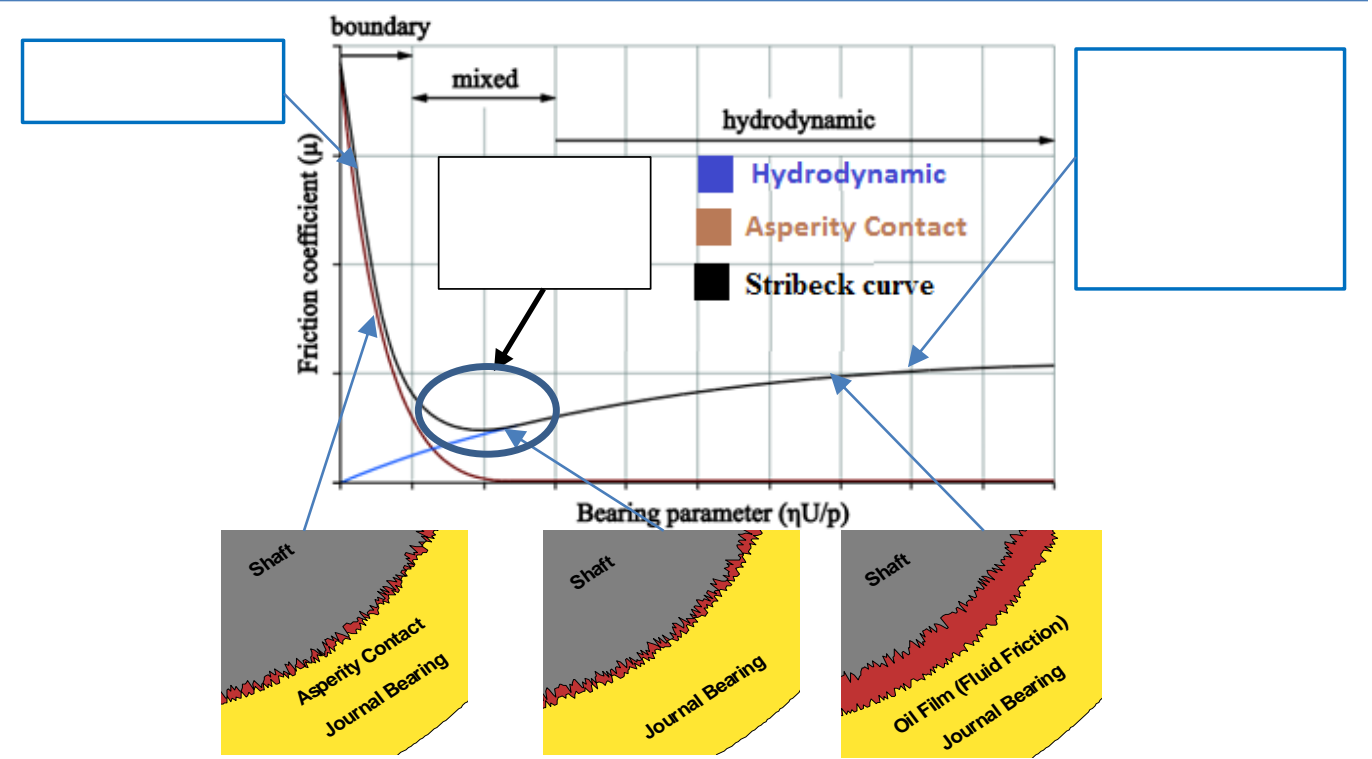

Figure 6: Lubrication regimes of a journal bearing [3]

\section{Vibration of Journal Bearing}

Vibration responses of a self-aligning journal bearing directly linked to the radial load, rotating speed, lubricant viscosity, oil film thickness, eccentricity, surface asperity characteristics and material of the bearing. Different forces placed on journal bearing affect the vibration responses and produce both low and high frequency vibrations. For example, the low journal whirling frequency is influenced by shaft fluctuating. Likewise, high random frequency bands of the vibration signals are occurred by asperity collisions and churns [5]. At hydrodynamic regime, journal bearing vibration sources often are generated by mechanical contact between the rotating shaft and stationary bearing [6]. High clearance causes looseness of bearing which generates more of a square wave than a sinusoid wave [7] and, many harmonics are generated from these signals. In the case of severe looseness, it is stretched all the way across the spectrum and half-harmonics are even generated in extreme cases (one-time, double times, three times, four times, five times, six times, etc.) rpm [7]. Another type of excitations is mechanical unbalance which causes a pure sinusoid and therefore generates a peak at one time rpm [8]. Journal bearing is often generate vibration peaks at frequencies lower than one time rpm. Another problem of the journal bearing is oil whirl. In which oil whirl is a phenomenon that vibrations are excited by oil film between frequency from 0.38 time rpm to 0.48 time rpm. Changes in viscosity and pressure of the oil and related loads can affect oil whirl [9]. Asperity collisions and churns interactions are sources of self-excitation, which result in high responses at structure resonances. The random high frequency of vibration responses is mainly related to two frictional effects. High frequency bands around $10 \mathrm{kHz}$ of the bearing are related with both asperity contacts and fluid friction through the method of clustering spectrum of vibration signals, [10]. The response frequency of self-excited vibration is very close to one of the system's natural frequencies [11]. Finally, the interaction between periodic responses and 
resonant responses produce modulation signals. The equation bellow presents different sources of vibration in a journal bearing [12].

$$
\begin{aligned}
& m_{s} \ddot{x}+\overbrace{k[p(x), \omega, \mu] x}^{\text {Hydrodynamic }}+\overbrace{\sum_{\mathrm{i}=1}^{\mathrm{n}} k_{\mathrm{i}} \Gamma[p(x), \omega, \mu] x}^{\text {Asperity churns }}+\overbrace{\sum_{\mathrm{i}=1}^{\mathrm{n}} k_{i} \Psi[p(x), \omega, \mu] x}^{\text {Asperity collisions }} \\
& =F_{r}+\overbrace{\sum_{\mathrm{i}}^{\mathrm{n}} \mathrm{A}_{\mathrm{i}} \cos \left(i \omega t+\alpha_{\mathrm{i}}\right)}^{\text {Unbalance+ }+ \text { misalignment }}
\end{aligned}
$$

where $m_{s}$ is the mass of the shaft; $k_{\mathrm{i}}$ denotes the bending stiffness of an arbitrary micro asperity; $k$ is the stiffness coefficients due to hydrodynamic pressure effect which includes inherent surface defects and journal elastic deformations of micro asperities and main load zones.

\section{Modulation Signal Bispectrum}

Understandably, high forces of external and internal excitation will generate massive vibration responses. Any mechanical problem will produce vibration with low frequency correlated to shaft rotational speed. Contrast, random asperity collisions will produce high band frequency. Due to a low frequency (information signal) is superimposed on a high frequency (carrier signal) modulation signal of vibration will be generated in journal bearing. Bispectrum is a non-linearity signal generated by interact two waves. MSB is used to detect coupling signal between shaft frequencies and the wideband compounds. The modulation signal of vibration is formed by nonlinear of two components, periodic and random signal. Thus, it is anticipated that bispectrum can give a more accurate representation of the vibration signal for misoperating diagnosis [13]. The Discrete Fourier Transform (DFT) $X(f)$ of a vibration signal $x(t)$ is defined in the form of Modulation Signal Bispectrum (MSB),

$$
B_{M S}\left(f_{x}, f_{c}\right)=E\left\langle X\left(f_{c}+f_{x}\right) X\left(f_{c}-f_{x}\right) X^{*}\left(f_{c}\right) X^{*}\left(f_{c}\right)\right\rangle
$$

The phase relationship of MSB is

$\varphi_{M S}\left(f_{x}, f_{c}\right)=\varphi\left(f_{c}+f_{x}\right)+\varphi\left(f_{c}-f_{x}\right)-\varphi\left(f_{c}\right)-\varphi\left(f_{c}\right)$

$=\varphi\left(f_{x}\right)+\varphi\left(-f_{x}\right)$

where $f_{x}$ is information frequency; $f_{c}$ is the carrier frequency, $f_{c}+f_{x}$ and $f_{c}-f_{x}$ are the higher and lower sideband frequencies respectively. It takes into account both $f_{c}+f_{x}$ and $f_{c}-f_{x}$ simultaneously in Equation above for quantifying the nonlinear effects of modulation signals. If they are due to the modulation effect between $f_{c}$ and $f_{x}$, a bispectral peak will be 
Monitoring Mis-Operating Conditions of Journal Bearings based on Modulation Signal Bispectrum Analysis of Vibration Signals

at bifrequency $B_{M S}\left(f_{x}, f_{c}\right)$. On the other hand, if these components such as various noises are not coupled but have random distribution, their magnitude of MSB will be close to zeros. In this way, the wideband noise and aperiodic components of vibration signals can be suppressed effectively so that the discrete components relating modulation effects can be represented sparsely and characterised more accurately.

A normalized form of MSB, also named as modulated signal bicoherence, is introduced as,

$$
b^{2}{ }_{M S}\left(f_{x}, f_{c}\right)=\frac{\left|B_{M S}\left(f_{x}, f_{c}\right)\right|^{2}}{E\left\langle\left|X\left(f_{c}\right) X\left(f_{c}\right) X^{*}\left(f_{c}\right) X^{*}\left(f_{c}\right)\right|^{2}\right\rangle E\left\langle\left|X\left(f_{c}+f_{x}\right) X\left(f_{c}-f_{x}\right)\right|^{2}\right\rangle}
$$

It is to measure the degree of coupling between three components against noise influences, in the same way as the conventional bicoherence $[14,15]$.

\section{Experimental Procedure}

A self-aligning spherical journal bearing, SA35M shown in Figure 7, has been tested. In this experimental, three types of lubrication are used to generate different operating conditions denoted as lube 22, 37 and 46 VG. Also, three different speed 1500, 1200 and $900 \mathrm{rpm}$ are used. Furthermore, four different radial loads 1, 5, 10 and 20 bar are exerted on the shaft supported between two bearings. These different viscosities, rotation speed and radial load will lead journal bearing to work under different lubricant regimes. Figure 7 shows the journal bearing test rig. An accelerometer sensor is fixed horizontally to collect the vibration signals. Also, an encoder and a pressure sensor are placed to measure the output rotating speed and radial load, respectively.
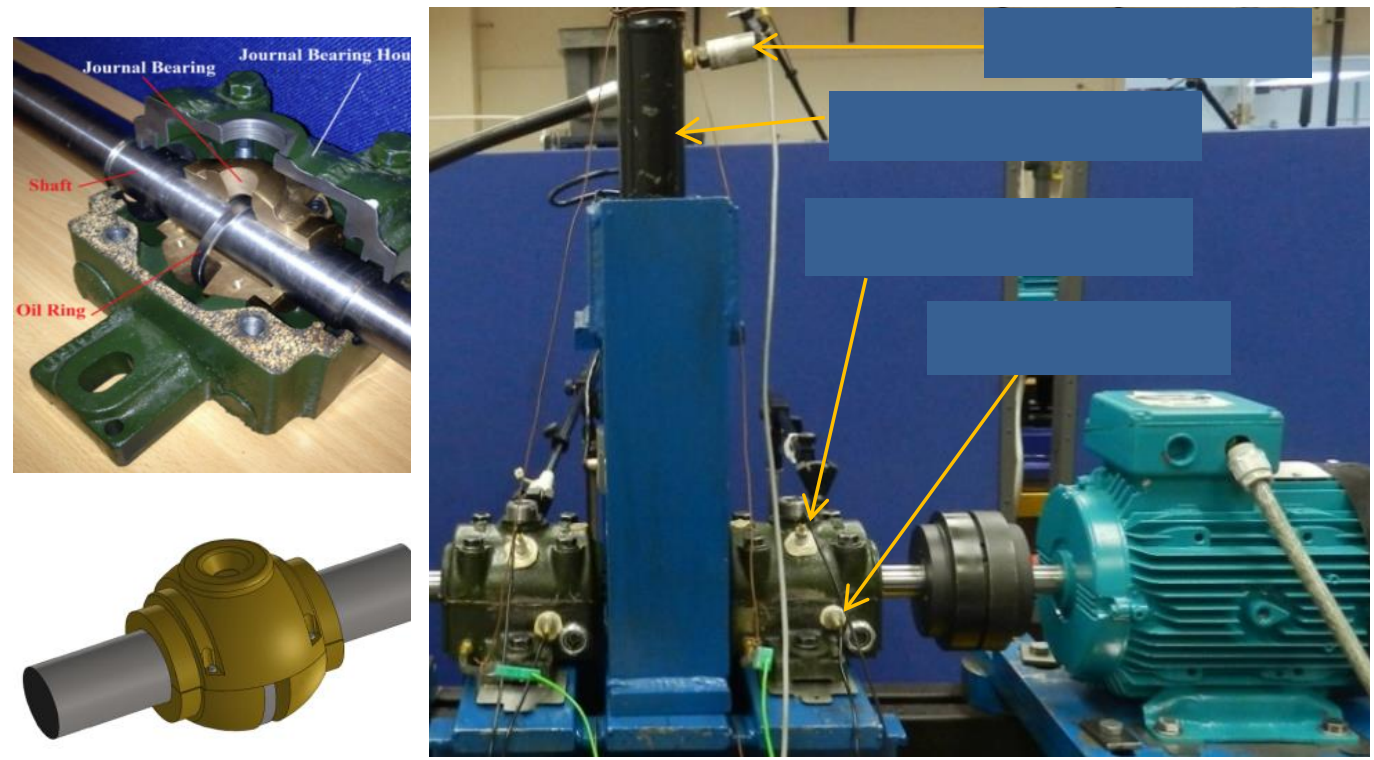

Figure 7: Self-aligning journal bearing components and test rig [12] 


\section{Experimental Results}

RMS values are proportional to speeds, loads but do not show any significant difference between lubrication types because of instability distribution of the oil by oil ring and nonlinearity distribution of the temperature. In theory, low speed causes more friction especially at high load but in the RMS results, Figure 8, show that the high speed always has high vibration and high load conditions generate high vibration energy. Also, different viscosities generate nonlinear RMS values of the vibration signals Thus in this study; the RMS values do not provide any good indication to present the bearing condition at different lubricant regimes. Therefore, these values do not consider to be a good indicator to obtain optimum operating conditions because they are always proportional to loads and speeds.
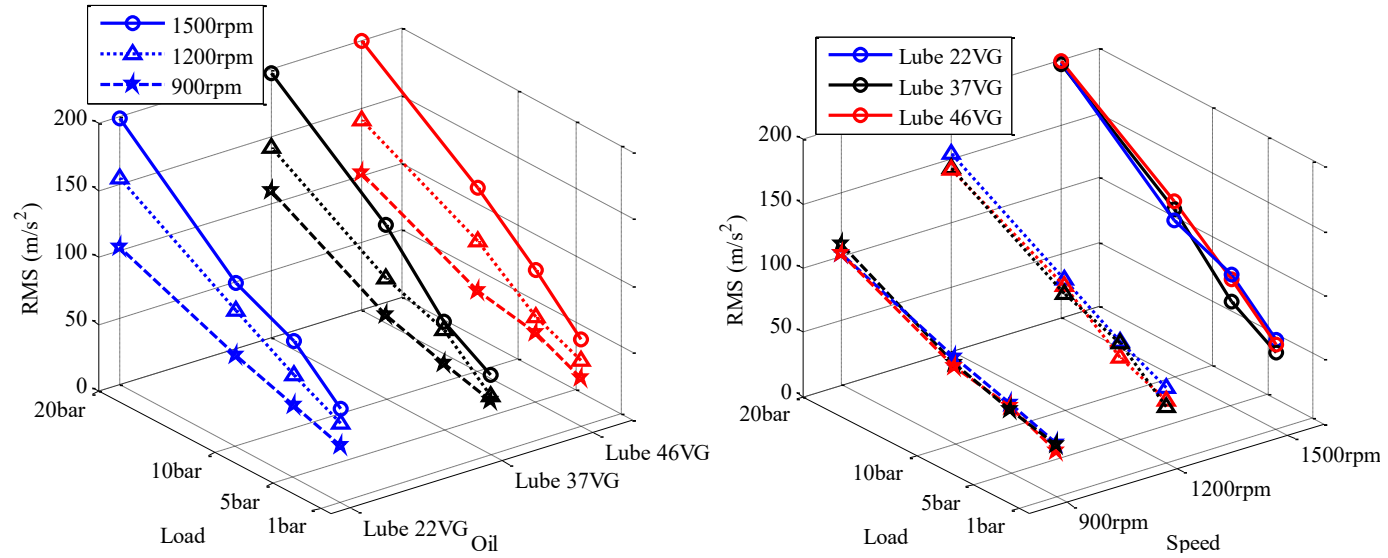

Figure 8: RMS values of vibration signals under different operating conditions

\subsection{MSB Magnitude and MSB Coherence Results}

Modulation signal bispectrum is dealing with coupled signals. In this manner, the wideband noise and periodic components of vibration signals can be suppressed effectively so that the discrete components relating modulation effects can be represented sparsely and characterised more accurately. MSB-Mag. and MSB-Coh. can provide a more clear representation of the journal bearing operation under different operating conditions. From Figure 10, it can be found that there is a high frequency band sensitive to changing radial load in MSB-Mag. These bands might be correlated with the friction of asperity collision caused by increasing the radial loads, which decrease the contact point at hmin and leads to metal-to-metal contact. In MSBCoh. Figure 12, there is clear frequency band related to shaft speed. As a result of that, the small wedge may decrease fluctuating of the shaft and make it rotates more stable. High viscosity lubricant has high damper coefficient to absorb more vibration than low viscosity, but it loses its ability to move easily into the gap between surfaces. Moreover, high viscosity might cause asperity churns at high radial clearance in load zone.

Proceedings of First Conference for Engineering Sciences and Technology (CEST-2018), vol. 2 
Monitoring Mis-Operating Conditions of Journal Bearings based on Modulation Signal Bispectrum Analysis of Vibration Signals

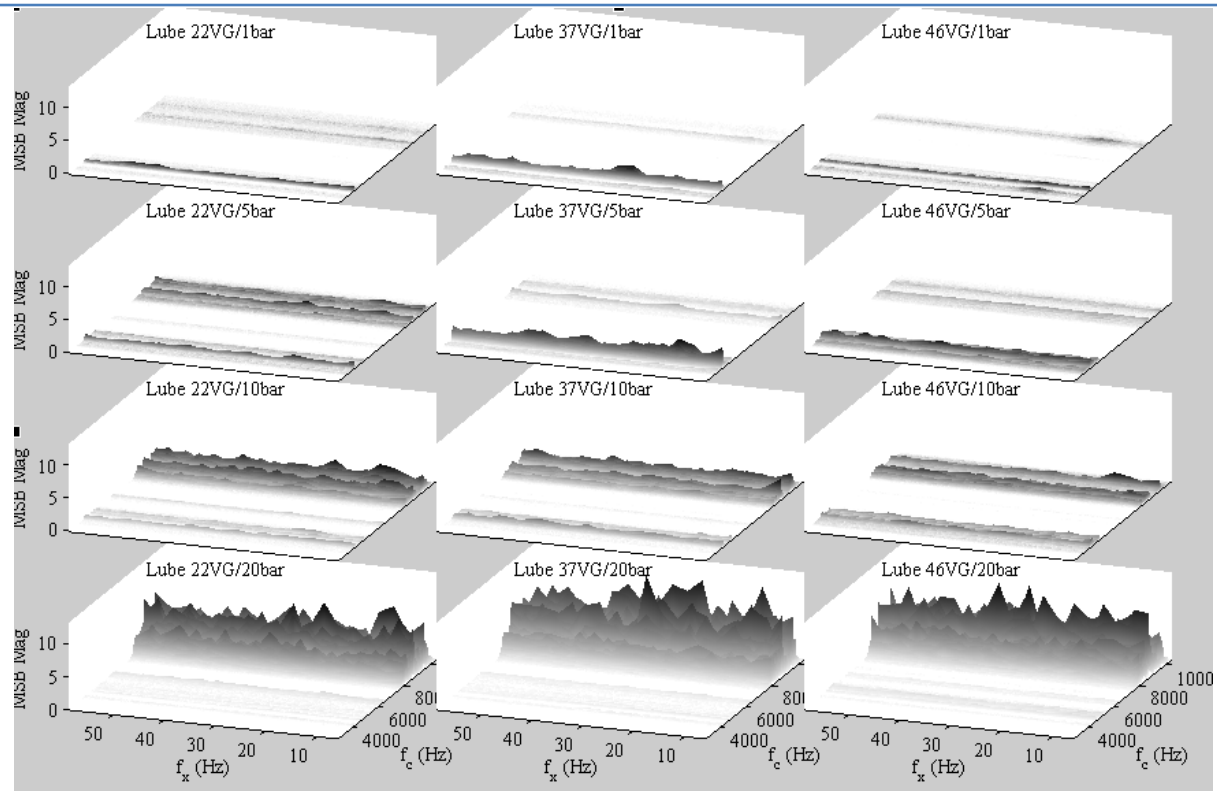

Figure 9: MSB-Mag. at 900rpm under different loads and viscosities

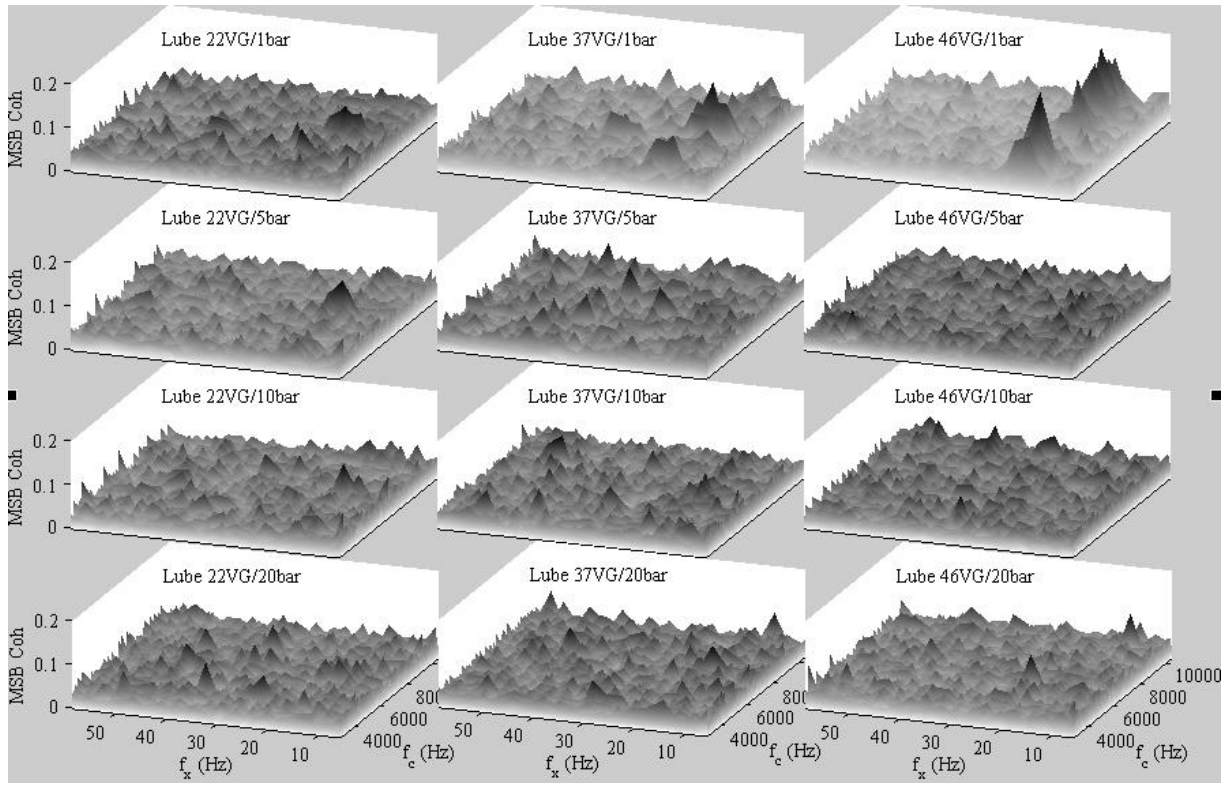

Figure 10: MSB-Coh. at 900rpm under different loads and viscosities

\subsection{Mean Values of MSB Magnitude and MSB Coherence Results}

MSB-Mag. manly presents asperity collision. In contrast, MSB-Coh. presents stability of the shaft. By calculating mean values of both MSB-Mag. and Coh. It can be found that the low load has the lowest mean of MSB-Mag., and at the same time has a higher mean value of MSBCoh. In contrast, high load has less value of MSB-Coh. and high value of MSB Mag. as can be seen in Figure 11 That means, high load makes shaft more stable but causes asperity collision. 
Hassin et al., CEST-2018, AIJR Proceedings 4, pp.509-517, 2018
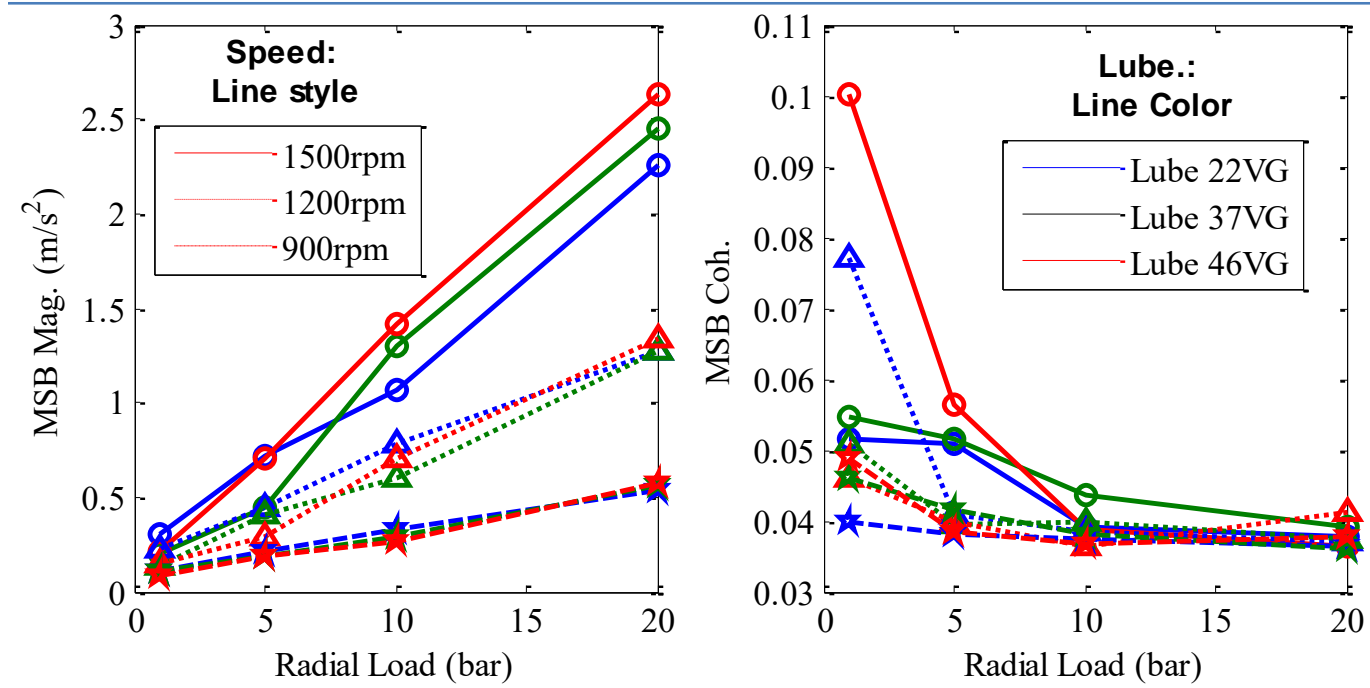

Figure 11: Mean values of MSB-Mag. and MSB-Coh.

Figure 12 shows gathering results of mean values of normalized MSB-Mag. and mean values of MSB-Coh. Examine the Figure representation; this operating condition could be considered as an acceptable operating conditions of the journal bearing compared with Stribeck curve in Figure 6. So, less mean value of MSB-Mag. and less value of MSB-Coh can be identified as an optimum operating condition.

Normalising MSB Mag. + MSB Coh

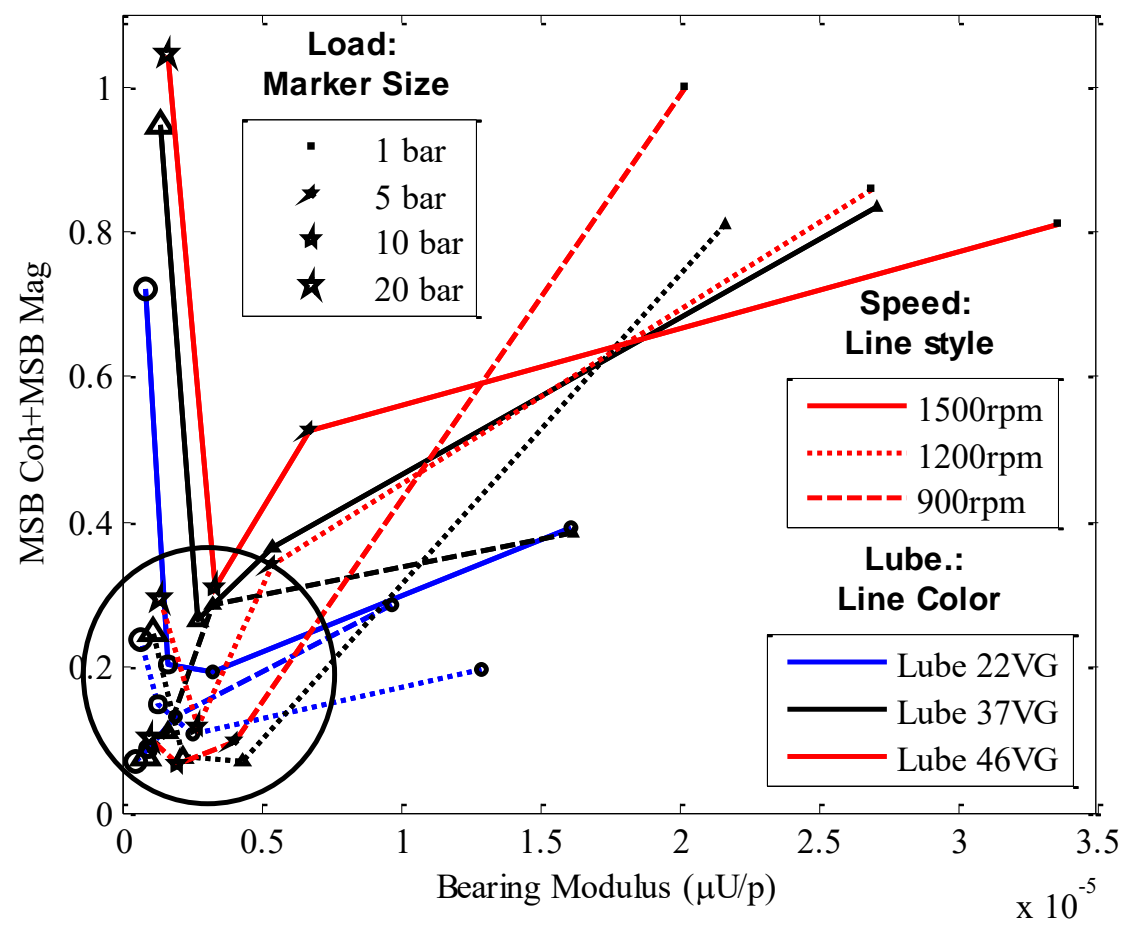

Figure 12: Representative Stribeck curve by coupling MSB-Mag. and MSB-Coh.

Proceedings of First Conference for Engineering Sciences and Technology (CEST-2018), vol. 2 
Monitoring Mis-Operating Conditions of Journal Bearings based on Modulation Signal Bispectrum Analysis of Vibration Signals

The optimum operating conditions in this experiment is at speed $1200 \mathrm{rpm}$ and under 5 bar load of oil 37. The worst operating condition at hydrodynamic regime is at speed 1500rpm under load 1 bar and oil 46. The worst operating condition at boundary regime is at speed 900 rpm under load 20 bar and oil 22. These results prove the theory of Stribeck curve.

\section{Conclusion}

When the shaft rotates under a different operating condition, vibration signals manifests rich frequency components due to various vibration excitations such as mechanical problem, asperity collisions and asperity-fluid interactions. The mechanical problem, unbalance, occurs when the radial clearance is too large. To avoid this instability, a small radial load should be applied, or radial clearance should be decreased. However, if the radial load exceeds a certain level, metal-to-metal contacts will occur and generate more unneeded frictions, so the radial load should be carefully chosen.

RMS values of raw data are not convenient to obtain optimum operating conditions and do not differentiate between speeds and oil viscosities under different loads. On the other hand, mean values of MSB-Mag. and MSB-Coh. are found to be effective to determine the optimum operating condition. Abnormal conditions such as high loads, low viscosities and low speeds have high mean values of MSB-Mag. In the meantime, un-wanted operating conditions such as low load, high viscosity and high speed have high means value of MSB-Coh. Finally the mis-operating conditions have been found by analysing vibration signals based on coupling MSB-Mag. and MSB-Coh.

\section{References}

[1] Sethiya, S., Condition Based Maintenance (CBM). Secy. to CME/WCR/JBP, 2006.

[2] Raharjo, P., An Investigation of Surface Vibration, Airbourne Sound and Acoustic Emission Characteristics of a Journal Bearing for Early Fault Detection and Diagnosis. University of Huddersfield, May 2013.

[3] Tomanik, E. and A. Ferrarese. Low friction ring pack for gasoline engines. in ASME 2006 Internal Combustion Engine Division Fall Technical Conference. 2006. American Society of Mechanical Engineers.

[4] Frene, J., et al., Hydrodynamic lubrication: bearings and thrust bearings. Vol. 33. 1997: Elsevier.

[5] Raharjo, P., et al., Vibro-Acoustic Characteristic of A Self Aligning Spherical Journal Bearing due to Eccentric Bore Fault. 2012.

[6] Kim, M., et al., Experimental identification of abnormal noise and vibration in a high-speed polygon mirror scanner motor due to mechanical contact of plain journal bearing. Microsystem technologies, 2010. 16(1-2): p. 3-8.

[7] Looseness - Bearing - VibrationSchool.com. 17 Nov 2015

[8] Unbalance - Single Plane - VibrationSchool.com. 11 Nov 2015.

[9] Journal bearing problem . VibrationSchool.com. 27 Nov 2015

[10] Osama Hassin, et al., Journal bearing lubrication monitoring based on spectrum cluster analysis of vibration signals in CMADEM 2015. 2015.

[11] Muszynska, A., Free, forced, and self-excited vibration. December 1997.

[12] Osama Hassin, Condition Monitoring of Journal Bearings for Predictive Maintenance Management Based on High Frequency Vibration Analysis, $\mathrm{PhD}$ thesis, May 2017.

[13] $\mathrm{Gu}, \mathrm{F}$., et al., Electrical motor current signal analysis using a modified bispectrum for fault diagnosis of downstream mechanical equipment. Mechanical Systems and Signal Processing, 2011. 25(1): p. 360-372.

[14] Gu, F., et al., A new method of accurate broken rotor bar diagnosis based on modulation signal bispectrum analysis of motor current signals. Mechanical Systems and Signal Processing, 2015. 50: p. 400-413.

[15] Gu, F., et al., A Novel Method for the Fault Diagnosis of a Planetary Gearbox based on Residual Sidebands from Modulation Signal Bispectrum Analysis. COMADEM 2014 16th-18th Sep. Brisbane, Australia., 2014. 